\title{
Chemistry and Immunomodulatory Activity of Frankincense Oil
}

Botros R. Mikhaeil*, Galal T. Maatooq, Farid A. Badria, and

Mohamed M. A. Amer

Department of Pharmacognosy, Faculty of Pharmacy, Mansoura University, Mansoura 35516, Egypt. E-mail: botros113426@yahoo.com.

* Author for correspondence and reprint requests

Z. Naturforsch. 58c, 230-238 (2003); received July 31/November 4, 2002

The yield of steam distillation of frankincense essential oil (3\%); and its physicochemical constants were determined. Capillary GC/MS technique was used for the analysis of the oil. Several oil components were identified based upon comparison of their mass spectral data with those of reference compounds published in literature or stored in a computer library. The oil was found to contain monoterpenes $(13.1 \%)$, sesquiterpenes $(1 \%)$, and diterpenes $(42.5 \%)$. The major components of the oil were duva-3,9,13-trien-1,5 $\alpha$-diol-1-acetate $(21.4 \%)$, octyl acetate $(13.4 \%)$, o-methyl anisole $(7.6 \%)$, naphthalene decahydro-1,1,4a-trimethyl-6-methylene-5-(3-methyl-2-pentenyl) (5.7\%), thunbergol $(4.1 \%)$, phenanthrene-7ethenyl-1,2,3,4,4a,5,6,7,8,9,10,10a-dodecahydro-1,1,4a,7-tetramethyl $(4.1 \%), \alpha$-pinene $(3.1 \%)$, sclarene $(2.9 \%)$, 9-cis-retinal $(2.8 \%)$, octyl formate $(1.4 \%)$, verticiol $(1.2 \%)$ decyl acetate $(1.2 \%), n$-octanol $(1.1 \%)$. The chemical profile of the oil is considered as a chemotaxonomical marker that confirmed the botanical and geographical source of the resin. Biologically, the oil exhibited a strong immunostimulant activity ( $90 \%$ lymphocyte transformation) when assessed by a lymphocyte proliferation assay.

Key words: Frankincense Essential Oil, Immunomodulatory Activity

\section{Introduction}

Frankincense oleogum resin is known as Olibanum, Luban Dakar, Bakhor or Kendar (In Arabic), Salai Guggal (in ayurvedic medicine). It is obtained by incision of the bark of several species of Boswellia, Burseraceae (Wallis, 1967; Evans, 1996). The plant is native to India, Arabian Peninsula (Yemen, Sultanate of Oman), Red Sea region of North-East Africa (Somalia, Eritrea) (Dietrich, 1900; Leung and Foster, 1996; Maupetit, 1984; Tschrich and Stock, 1935). Several commercial brands of the resin are available; the Aden and Eritrean brands are usually regarded as derived from Boswellia carterii Birdw., and B. frereana Birdwood, respectively. Sudanese olibanum is obtained from Boswellia papyrifera Del. whereas Indian olibanum is derived mainly from Boswellia serrata Roxb. (Verghese, 1988). There has been a considerable work done on the composition of olibanum oil from different species and commercial brands of Boswellia (Abdel wahab et al., 1987; Ammar et al., 1994; Guenther, 1972; Obermann, 1978; Strappaghetti et al., 1982). The chemical profile of the oil can be used as a chemotaxonomical marker to distinguish between the different com- mercial varieties of frankincense. The composition of the oil differs according to the climate, harvest conditions, and geographical source (Yates and Wenninger, 1970). Accordingly, frankincense can be classified into country of origin by identifying certain constituents in the essential oil using GC/ MS technique (Ammar et al., 1994; Hayashi et al., 1998; Strappaghetti et al., 1982; Yates and Wenninger, 1970). The main constituent of Aden and Omani oil is $\alpha$-pinene (43\%) whereas, Eritrean, Turkish oils are rich in octyl acetate (52\%). The Indian oil is rich in $\alpha$-thujene $(61 \%)$. The taxonomic origin of Omani, Aden oil is B. frereana Birdwood, while that of Eritrean and Turkish is $B$. carterii Birdw. The Indian brand comes from $B$. serrata Roxb (Hayashi et al., 1998; Verghese, 1988). The essential oil of frankincense is one of the most commonly used oils in aromatherapy practice nowadays. The oil is very good for respiration, eases breathing and is therefore useful for asthma patients. It has a soothing action in colds, cough, bronchitis and laryngitis (Lawless, 1996). It has excellent actions on skin for removing scars and stretch marks (Ryman, 1997). Frankincense oil produced contraction of phrenic-nerve diaphragm muscle (skeletal muscle) and inhibition of 
twitch response to nerve stimulation and exhibited a spasmogenic effect on smooth muscle in vitro. These effects may be due to post-junctional block of neuromuscular transmission and an action on the sarcoplasmic reticulum to increase intracellular calcium (Maria and Stephen, 1997). The oil exhibited anti-bacterial and antifungal activities (Abdel wahab et al., 1987; Gangwal and Vardhan, 1995). Most of the previous reports investigated the anti-inflammatory, immunomodulatory, and anti-leukotriene activity of the resin and especially its major components, boswellic acid derivatives (Ammon et al., 1991; Gupta et al., 1997, 1998; Safayhi et al., 1992, 1995; Sharma et al., 1988; Singh and Atal, 1986), so it was deemed of interest to investigate the chemistry, and immunomodulatory activity of the essential oil.

\section{Results and Discussion}

The yield of volatile oil of Frankincense obtained by steam distillation of the finely powdered oleogum resin derived from Boswellia carterii Birdw. was $3 \%$. The oil is pale yellow with agreeable balsamic slightly spicy and lemony odor. The oil possesses the following physicochemical constants: specific gravity $\left(25^{\circ} \mathrm{C}\right), 0.875$; refractive in$\operatorname{dex}\left(20^{\circ} \mathrm{C}\right), 1.446$; optical rotation $[\alpha]_{\mathrm{D}}^{20^{\circ}}:-1.11$ ( $1 \%$ solution in $95 \%$ ethanol); solubility $1: 1 \mathrm{w} / \mathrm{v}$ in ethanol $95 \%$ and $1: 2 \mathrm{w} / \mathrm{v}$ in ethanol $70 \%$; acid value, 12.05; ester value and ester percentage (calculated as octyl acetate) are 127.46 and 39\%, respectively; and the saponification value is 139.51 .

The GC/MS chromatogram of the steam distilled oil revealed the presence of several components (Fig. 1; Table I) that were identified through comparison of the fragmentation patterns in the resulting mass spectra with those published in literature (Adams, 1989, 1995) and using NST mass spectral data base of the gas chromatograph computer.

The volatile oil contains a high proportion of esters (ca. $40.06 \%)$ of which duva-3,9,13-trien-1,5 $\alpha$ diol-1-acetate is the major component $(21.35 \%)$, octyl acetate $(13.39 \%)$, octyl formate $(1.41 \%)$, bornyl acetate $(0.09 \%)$, citronellyl acetate $(0.74 \%)$, neryl acetate $(0.48 \%)$, geranyl acetate $(0.62 \%)$, hexyl $n$-hexanoate $(0.09 \%)$, decyl acetate $(1.16 \%)$, farnesyl acetate (E,E) $(0.01 \%)$, benzyl benzoate $(0.22 \%)$, and duva-3,9,13-trien-1 $\alpha$-ol-5,8-oxide-1acetate $(0.5 \%)$.
The oil contains several monoterpene hydrocarbons such as; $\beta$-pinene $(0.25 \%), \alpha$-pinene $(3.11 \%)$, isoterpinolene $(0.04 \%), \alpha$-phellandrene $(0.03 \%), \beta$ phellandrene $(0.19 \%)$, sabinene $(0.22 \%), \beta$-myrcene $(0.2 \%), d$-limonene $(7.6 \%)$, and cis-ocimene $(0.37 \%)$; monoterpene alcohols viz. $\beta$-citronellol $(0.2 \%)$, cis-carveol $(0.04 \%), \quad$ isopinocampheol $(0.12 \%)$, trans-terpin $(0.46 \%)$; monoterpene ketones viz. carvone $(0.26 \%)$, and piperitone $(0.03 \%)$.

Unlike a previous analysis (Ammar et al.,1994), that reported the absence of sesquiterpenoids in the essential oil of Boswellia carterii Birdwood; several sesquiterpenes were detected in the investigated oil viz. $\alpha$-copaene $(0.35 \%), \delta$-selinene $(0.24 \%)$, maaliane $(0.02 \%)$, viridiflorol $(0.06 \%), \alpha$-muurolol $(0.03 \%), \beta$ bisabolene $(0.15 \%)$, cis-calamenene $(0.01 \%)$, spathulenol $(0.03 \%)$, and cis-nerolidol $(0.07 \%)$.

Several diterpenes were detected in the oil especially those of the cembranoid skeleton such as, the previously reported cembrene $(0.27 \%)$, isocembrene $(0.28 \%)$ (Ohloff, 1994), in addition, verticiol (1.22\%), duva-4,8,13-trien-1,3 $\alpha$-diol (0.23\%), thunbergol (4.07\%), duva-3,9,13-trien-1,5 $\alpha$-diol $(0.06 \%)$, duva-3,9,13-trien-1 $\alpha$-ol-5,8-oxide-1-acetate $(0.5 \%)$, duva-3,9,13-trien-1,5 $\alpha$-diol-1-acetate

$(21.35 \%)$ are detected for the first time in olibanum essential oil. The presence of such high percent of cembranoid diterpenes in the essential oil of frankincense derived from Boswellia carterii Birdwood is reported for the first time. This rare and unique type of diterpenes has a 14-membered monocyclic ring and is characteristic for marine organisms such as soft corals (Rashid et al., 2000) and also detected in tobacco (Eklund et al., 1992). Diterpenes of other classes that were also detected for the first time in olibanum oil include, isophyllocladene (kaur-15-ene) (0.58\%), phenanthrene-7-ethenyl-1,2, 3,4,4a,5,6,7,8,9,10,10a-dodecahydro-1,1,4a,7-tetramethyl (4.06\%), beyerene $(0.96 \%)$, sclarene $(2.88 \%)$, and naphthalene decahydro-1,1,4a-trimethyl-6-methylene-5-(3-methyl-2-pentenyl) (5.7\%).

These results suggested that our sample of the frankincense oleogum resin is close to the Eritrean brand with octyl acetate being the major component $(13.39 \%)$. The complete absence of verbenone and the low percent of $\alpha$-pinene $(3.11 \%)$ excluded the Aden variety while, lacking $\alpha$-thujene precludes the Indian brand.

Lymphocyte proliferation (mitogenesis) assay involves study of a specific immune response. The as- 
Table I. Results of GC/MS analysis of the essential oil of frankincense oleogum resin.

\begin{tabular}{|c|c|c|c|c|c|c|c|}
\hline $\begin{array}{l}\text { Peak } \\
\text { scan \# }\end{array}$ & $\begin{array}{c}\text { Composition } \\
\%\end{array}$ & $\begin{array}{l}\text { Retention } \\
\text { Time }\left(t_{R}\right) \\
{[\text { min }]}\end{array}$ & $\begin{array}{c}\mathrm{M}^{+} \\
\text {peak }\end{array}$ & $\begin{array}{l}\text { Base } \\
\text { peak }\end{array}$ & $\begin{array}{l}\text { Major peaks } \\
\qquad(\mathrm{m} / \mathrm{z})\end{array}$ & Component & $\begin{array}{l}\text { Adams } \\
(1995) \\
\text { DB-5* }\end{array}$ \\
\hline 302 & 0.25 & $5: 46$ & 136 & 93 & $40.3,55,67,79,107,121$ & $\beta$-pinene & 386 \\
\hline 361 & 3.11 & $6: 05$ & 136 & 93 & $41,53.2,67,77.1,91.1,105$ & $\alpha$-pinene & 319 \\
\hline 410 & 0.04 & $6: 30$ & 134 & 93.1 & $40.3,53,67.2,91.1,121$ & isoterpinolene & 602 \\
\hline 424 & 0.03 & $6: 37$ & 136 & 93 & $40.3,53,65.2,77.2,91,119$ & $\alpha$-phellandrene & 435 \\
\hline 500 & 0.19 & $7: 15$ & 136 & 93 & $41.2,53,69.2,77.1,91,121$ & $\beta$-phellandrene & 482 \\
\hline 519 & 0.22 & $7: 25$ & 136 & 93 & $41.2,53,69,83,121.1$ & sabinene & 379 \\
\hline 572 & 0.2 & $7: 51$ & 136 & 93 & $41.2,53,69.2,79,109$ & $\beta$-myrcene & 408 \\
\hline 664 & 0.18 & $8: 37$ & 122 & 122.1 & 41.2,51.1,77.1,91,107 & $o$-methyl anisole & 459 \\
\hline 799 & 7.6 & $9: 45$ & 136 & 67 & $41,53,67,79,93,121$ & $d$-limonene & 481 \\
\hline 849 & 0.37 & $10: 10$ & 136 & 93.1 & $41,53,67,79,91,105,121,136$ & cis-ocimene & 498 \\
\hline $\begin{array}{l}1065 \\
1117\end{array}$ & 1.08 & $11: 59$ & 130 & 41 & $55,69,83,97.2,112$ & n-octanol & 564 \\
\hline $\begin{array}{l}\text { To } \\
1418 \\
1755\end{array}$ & 1.41 & $13: 40$ & 158 & 56 & $42,70.1,84,93,121$ & octyl formate & - \\
\hline $\begin{array}{l}\text { To } \\
2051\end{array}$ & 13.39 & $18: 56$ & 172 & 43 & $41,56,61,70,84,112,116$ & octyl acetate & - \\
\hline 2064 & 0.2 & $20: 21$ & 156 & 41 & $55,60,69,73,84,95,105,123,138$ & $\beta$-citronellol & 950 \\
\hline 2077 & 0.04 & $20: 27$ & 152 & 41 & $55,59,67,69,81,84,109,119,134$ & cis-carveol & 953 \\
\hline 2098 & 0.26 & $20: 38$ & 150 & 82 & $41,54,67,79,93,108,137,150$ & carvone & 984 \\
\hline 2121 & 0.03 & $20: 49$ & 150 & 82 & $41,55,69,82,95,110,137$ & piperitone & 1011 \\
\hline 2155 & 0.56 & 21:06 & 152 & 152 & $44,52,66,77,91,109,123$ & 2,6-dimethoxy toluene & - \\
\hline 2177 & 0.15 & $21: 17$ & 158 & 43 & $55,70,83,97,112,123,152$ & 1-decanol & 1063 \\
\hline 2188 & 0.12 & $21: 23$ & 154 & 43 & $55,69,83,94,107,112,152$ & isopinocampheol & 822 \\
\hline 2206 & 0.09 & $21: 32$ & 196 & 43 & $55,69,80,95,108,121,136,154$ & bornyl acetate & 1099 \\
\hline 2380 & 0.46 & $23: 04$ & 172 & 129 & $\begin{array}{c}41,, 43,55,60,83,87,97,101,115,129 \\
143\end{array}$ & trans-terpin & - \\
\hline 2489 & 0.74 & $23: 54$ & 198 & 43 & $41,55,67,69,81,95,109,123,138$ & citronellyl acetate & 1275 \\
\hline 2529 & 0.48 & $24: 14$ & 196 & 41 & $43,53,69,80,93,107,121,136,154$ & neryl acetate & 1303 \\
\hline 2588 & 0.35 & $24: 44$ & 204 & 161 & $41,43,55,67,81,93,105,119,133,161$ & $\alpha$-copaene & 1334 \\
\hline 2623 & 0.62 & 25:01 & 196 & 41 & $43,53,69,80,93,107,121,136,143$ & geranyl acetate & 1352 \\
\hline 2648 & 0.09 & $25: 14$ & 200 & 43 & $41,55,56,61,69,84,99,117$ & hexyl n-hexanoate & 1352 \\
\hline 2780 & 1.16 & $26: 20$ & 200 & 43 & $41,55,61,70,83,97,112,140,172$ & decyl acetate & 1420 \\
\hline 2908 & 0.24 & $27: 24$ & 204 & 43 & $\begin{array}{c}41,55,60,67,79,91,93,105,119,129 \\
133,147,161,172,189\end{array}$ & $\delta$-selinene & - \\
\hline 2964 & 0.02 & $27: 53$ & 206 & 43 & $\begin{array}{c}41,55,69,79,91,93,105,119,129,133 \\
147,161,189\end{array}$ & maaliane & - \\
\hline 3029 & 0.06 & $28: 25$ & 222 & 43 & $\begin{array}{c}41,55,67,71,81,95,105,109,119,121 \\
131,145,151,198,202\end{array}$ & viridiflorol & 1859 \\
\hline 3042 & 0.03 & $28: 32$ & 222 & 43 & $\begin{array}{c}55,67,79,81,91,93,105,119,133,135 \\
161,178,204\end{array}$ & $\alpha$ - muurolol & 1984 \\
\hline 3132 & 0.01 & $29: 17$ & 264 & 41 & $\begin{array}{c}55,69,81,93,107,119,121,137,148 \\
149,161,162,189\end{array}$ & farnesyl acetate $(\mathrm{E}, \mathrm{E})$ & 2419 \\
\hline 3205 & 0.15 & $29: 54$ & 204 & 41 & $\begin{array}{c}43,53,69,79,93,105,109,119,121,133 \\
147,161,189\end{array}$ & $\beta$-bisabolene & 1667 \\
\hline 3262 & 0.01 & $30: 22$ & 202 & 159 & $\begin{array}{c}41,43,55,65,65,69,77,81,91,105,115 \\
129,131\end{array}$ & cis-calamenene (1S) & 1695 \\
\hline 3286 & 0.03 & $30: 34$ & 220 & 43 & $\begin{array}{c}41,55,67,69,79,83,91,93,105,119,131 \\
147,159,177,187,205\end{array}$ & (-)-spathulenol & 1825 \\
\hline 3480 & 0.07 & $32: 12$ & 222 & 41 & $\begin{array}{c}43,55,69,81,93,107,121,136,148,161 \\
189,206\end{array}$ & cis-nerolidol & 1724 \\
\hline $\begin{array}{l}4455- \\
4466\end{array}$ & 0.22 & $40: 21$ & 212 & 105 & $43,51,65,77,91,112,145,167,194$ & benzyl benzoate & 2245 \\
\hline $\begin{array}{l}4558- \\
4671\end{array}$ & 1.11 & $41: 37$ & 270 & 43 & $\begin{array}{c}43,55,60,73,83,105,129,159 \\
185,199,228\end{array}$ & unknown & - \\
\hline 4729 & 0.27 & $42: 38$ & 272 & 159 & $\begin{array}{c}41,43,55,69,91,105,119,133,145,159 \\
187,204,229\end{array}$ & cembrene & 2597 \\
\hline 4795 & 0.28 & $43: 12$ & 272 & 159 & $\begin{array}{c}41,43,55,69,81,91,105,119,133,145 \\
159,161,187,204,229,257\end{array}$ & isocembrene & - \\
\hline 5000 & 0.58 & $44: 54$ & 272 & 43 & $\begin{array}{c}41,55,69,81,91,93,95,105,107,119 \\
121,136,147,161,173,189,203,228 \\
257,272\end{array}$ & $\begin{array}{l}\text { isophyllocladene } \\
\text { (kaur-15-ene) }\end{array}$ & 2664 \\
\hline
\end{tabular}


Table I (content).

\begin{tabular}{|c|c|c|c|c|c|c|c|}
\hline $\begin{array}{l}\text { Peak } \\
\text { scan \# }\end{array}$ & $\begin{array}{l}\text { Composition } \\
\%\end{array}$ & $\begin{array}{l}\text { Retention } \\
\text { Time }\left(t_{R}\right) \\
{[\mathrm{min}]}\end{array}$ & $\begin{array}{c}\mathrm{M}^{+} \\
\text {peak }\end{array}$ & $\begin{array}{l}\text { Base } \\
\text { peak }\end{array}$ & $\begin{array}{l}\text { Major peaks } \\
\qquad(m / z)\end{array}$ & Component & $\begin{array}{l}\text { Adams } \\
(1995) \\
\text { DB-5* }\end{array}$ \\
\hline $\begin{array}{l}5064- \\
5252\end{array}$ & 1.22 & $46: 35$ & 290 & 272 & $\begin{array}{c}41,43,55,69,81,91,93,107,119,121,133 \\
147,161,173,187,201,229,257,288\end{array}$ & verticiol & - \\
\hline 5305 & 4.06 & $47: 27$ & 272 & 93 & $\begin{array}{c}41,55,68,81,93,107,121,133,147,161 \\
189,201,215,229,257,272\end{array}$ & $\begin{array}{c}\text { phenanthrene-7- } \\
\text { ethenyl-1,2,3,4,4a,5,6,7, } \\
\text { 8,9,10,10a-dodeca- } \\
\text { hydro-1,1,4a,7- } \\
\text { tetramethyl }\end{array}$ & - \\
\hline 5346 & 0.96 & $47: 48$ & 272 & 41 & $\begin{array}{c}41,43,55,79,91,93,105,107,133,147 \\
159,174,186,201,215,227,255,272\end{array}$ & beyerene & 2587 \\
\hline $\begin{array}{l}5433- \\
5565\end{array}$ & 2.88 & 49:00 & 272 & 257 & $\begin{array}{c}41,55,67,79,91,93,107,121,133,147 \\
161,173,187,201,229,257,272\end{array}$ & sclarene & 2672 \\
\hline 5615 & 5.7 & $50: 03$ & 272 & 272 & $\begin{array}{l}41,55,67,79,91,107,119,121,147,161 \\
173,189,201,229,257,272\end{array}$ & $\begin{array}{l}\text { naphthalene decahydro- } \\
1,1,4 a-\text { trimethyl-6- } \\
\text { methylene-5-(3-methyl-2- } \\
\text { pentenyl) }\end{array}$ & - \\
\hline 5631 & 2.82 & $50: 11$ & 284 & 95 & $\begin{array}{c}41,55,67,79,91,105,119,134,147,151 \\
164,188,213,227,257,270,284\end{array}$ & 9-cis-retinal & - \\
\hline 5650 & 0.23 & $50: 20$ & 306 & 95 & $\begin{array}{c}\text { 41,43,55,67,82,95,107,121,135,137, } \\
149,159,177,189,213,227,256,288,304\end{array}$ & $\begin{array}{l}\text { duva-4,8,13-trien- } \\
1 \alpha, 3 \alpha \text {-diol }\end{array}$ & - \\
\hline 5691 & 4.07 & $50: 41$ & 290 & 177 & $\begin{array}{c}41,43,55,67,81,91,109,121,133,147 \\
161,177,189,227,257,272,288\end{array}$ & thunbergol & - \\
\hline 5774 & 0.06 & $51: 59$ & 306 & 43 & $\begin{array}{c}41,55,69,81,93,107,121,135,147,159 \\
187,203,206,245,288\end{array}$ & $\begin{array}{c}\text { duva-3,9,13-trien- } \\
1,5 \alpha \text {-diol }\end{array}$ & - \\
\hline 5889 & 0.5 & $52: 20$ & 346 & 43 & $\begin{array}{c}41,55,67,81,93,107,121,133,147,161 \\
175,187,203,215,229,243,272,303\end{array}$ & $\begin{array}{l}\text { duva-3,9,13-trien- } 1 \alpha \text { - } \\
\text { ol-5,8-oxide-1-acetate }\end{array}$ & - \\
\hline 6270 & 21.35 & $55: 31$ & 348 & 43 & $\begin{array}{c}41,55,71,81,93,107,125,136,151,156 \\
161,202,238,263,288,306,308,348\end{array}$ & $\begin{array}{l}\text { duva-3,9,13-trien- } \\
1,5 \alpha \text {-diol-1-acetate }\end{array}$ & - \\
\hline
\end{tabular}

* Elution time in seconds according to Adams (1995).

say investigates the mitogenic effect of the crude drug on T-lymphocytes proliferation. Anti-proliferative activity on T-lymphocyte culture indicates immunosuppression while promotion of T-lymphocyte proliferative response means immunostimulation (Nores et al., 1997; Puri et al., 1993; Sairam et al., 1997). The essential oil of frankincense $(100 \mu \mathrm{l} / \mathrm{ml})$ in DMSO induced a mitogenic response $(90 \%$ lymphocyte proliferation) that is comparable to standard well known immunostimulants e.g. Echinacea purpurea aqueous extract (95\%) and levamisole (85\%) (Fig. 2).

In conclusion, based on the chemical profile of the oil, our sample of frankincense oleogum resin is of the Eritrean brand which is derived from Boswellia carterii Birdw. The oil demonstrated immunostimulant activity which is an added value to the previously reported anti-inflammatory, immunomodulatory, and anti-leukotriene activity of the resin. This encourages the use of olibanum oleogum resin in several immune disorders.

\section{Experimental:}

\section{Plant material}

Frankincense (oleogum resin of Boswellia carterii Birdwood, Burseraceae, was purchased from the local market of herbs and spices in Egypt. It was authenticated by comparison with a genuine sample kept in the drug museum of Pharmacognosy Department, Faculty of Pharmacy, Cairo University.

\section{Reagents for lymphocyte transformation assay}

Heparinized peripheral venous blood was obtained from healthy volunteers at the blood bank of Mansoura University Hospital; Ficoll/Hypaque obtained from Amersham Pharmacia, Uppsala, Sweden; phytohaemagglutinin (PHA) obtained from Difco, Detroit, MI, USA; Hank's balanced salt solution (HBSS); foetal calf serum (FCS); glutamine; HEPES (N-2-Hydroxyethylpiperazine-N' 2-ethanesulfonic acid)-buffer and RPMI-1640 me- 


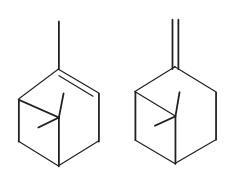<smiles>C=C1C=CC(=C(C)C)CC1</smiles><smiles>CC1=CCC(C(C)C)C=C1</smiles><smiles>C=C1C=CC(C(C)C)CC1</smiles>

$\alpha$-pinene $\beta$-pinene isoterpinolene $\alpha$-phellandrene $\beta$-phellandrene<smiles>COc1ccccc1C</smiles><smiles>C=C(C)C1CC=C(C)CC1</smiles><smiles>C=CC(C)=CCC=C(C)C</smiles>

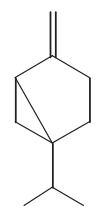

sabinene $\beta$-myrcene

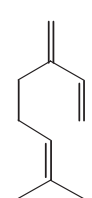

$o$-methyl anisole

$d$-limonene

cis-ocimene

$\mathrm{CH}_{3}-\left(\mathrm{CH}_{2}\right)_{7}-\mathrm{OH}$

$\mathrm{CH}_{3}-\left(\mathrm{CH}_{2}\right)_{7}-\mathrm{OCOOH}$

$\mathrm{CH}_{3}-\left(\mathrm{CH}_{2}\right)-\mathrm{OOCCH}_{3}$

octyl acetate<smiles>COc1cccc(OC)c1C</smiles>

2,6-dimethoxy toluene

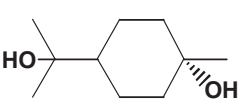

trans-terpin

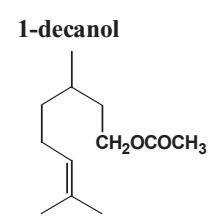

citronellyl acetate

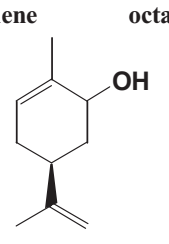

cis-carveol octanol<smiles>C=C(C)[C@H]1CC=C(C)C(=O)C1</smiles>

carvone

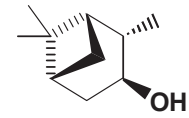

(

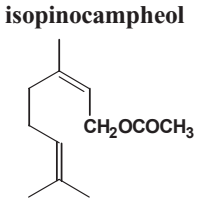

neryl acetate

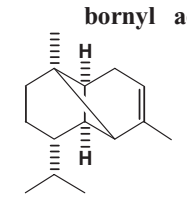

$\alpha$-copaene

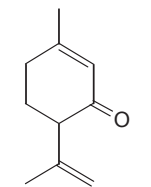

piperitone octyl formate<smiles>CC(=O)OCC=C(C)CCC=C(C)C</smiles>

$\mathrm{CH}_{3}\left(\mathrm{CH}_{2}\right)_{5} \mathrm{OCO}\left(\mathrm{CH}_{2}\right)_{4} \mathrm{CH}_{3}$

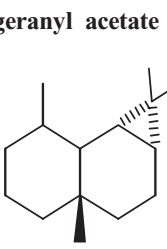

maaliane<smiles>C=C(CCC=C(C)C)C1CC=C(C)CC1</smiles>

$\beta$-bisabolene

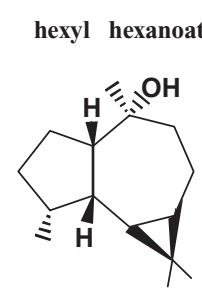

viridiflorol

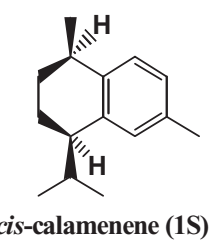

$\mathrm{CH}_{3}\left(\mathrm{CH}_{2}\right)_{9} \mathrm{OCOCH}_{3}$

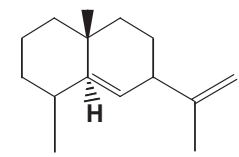

decyl acetate

$\delta$-selinene

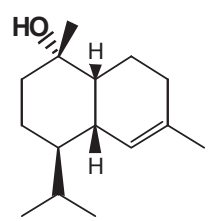

$\alpha$-muurolol

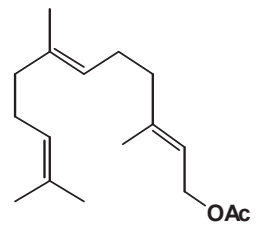

farnesyl acetate $(E, E)$
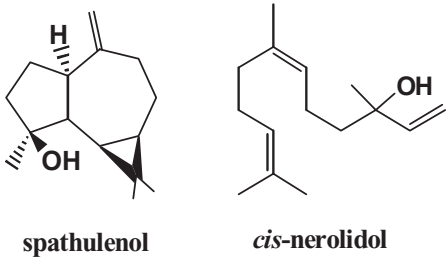

cis-nerolidol 
<smiles>O=C(OCc1ccccc1)c1ccccc1</smiles>

benzyl benzoate

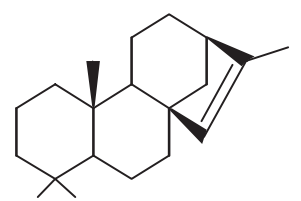

isophyllocladene (kaur-15-ene)

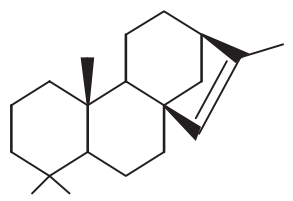

beyerene

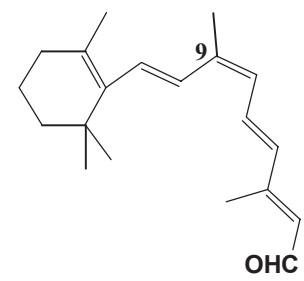

9-cis-retinal

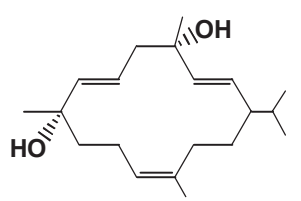

duva-3,9,13-triene $-1,5 \alpha$-diol

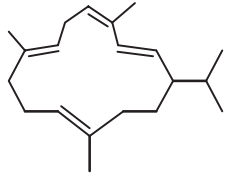

cembrene<smiles>C/C1=C/CC/C=C(/C)CC2C(CC1)C1CC[C@@](C)(O)C2C1</smiles>

verticiol<smiles>C=C1CC[C@H]2C(=C)CCC3C(C)(C)CCC[C@]32C1</smiles>

sclarene

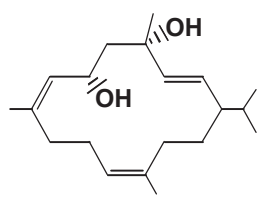

duva-4,8,13-triene $-1,3 \alpha$-diol

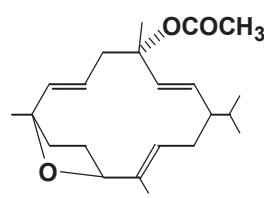

duva-3,9,13-triene-1 $\alpha$-hydroxy $-5,8-0 x i d e-1-a c e t a t e$

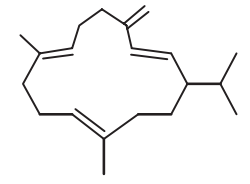

isocembrene<smiles>C=C[C@]1(C)CCC2=C(CCC3C(C)(C)CCC[C@@]23C)C1</smiles>

phenanthrene-7-ethenyl-1,2,3,4,

4a,5,6,7,8,9,10,10a-dodecahydro -1,1,4a,7-tetramethyl<smiles>C=C1CCC2C(C)(C)CCC[C@]23C[C@H]1CC=C(C)C3=C</smiles>

naphthalene decahydro $-1,1,4 a-t r i m e t h y l-6-m e t h y l e n e-$ 5-(3-methyl-2-pentenyl)

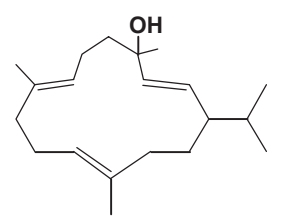

thunbergol

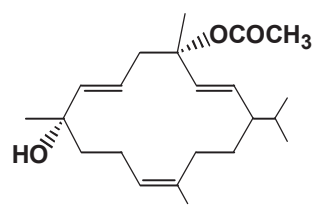

duva-3,9,13-triene $-1,5 \alpha$-diol-1-acetate

Fig. 1. Components of the essential oil of Boswellia carterii Birdwood.

dium obtained from Gibco BRL, Life Technologies, Pailsey, Scotland; crystalline penicillin G and streptomycin obtained from El-Nile Pharmaceutical Co., Cairo, Egypt.
Preparation of the volatile oil

Finely ground oleogum resin $(500 \mathrm{~g})$ was subjected to steam distillation using Clevenger's apparatus until complete exhaustion. The oil was col- 


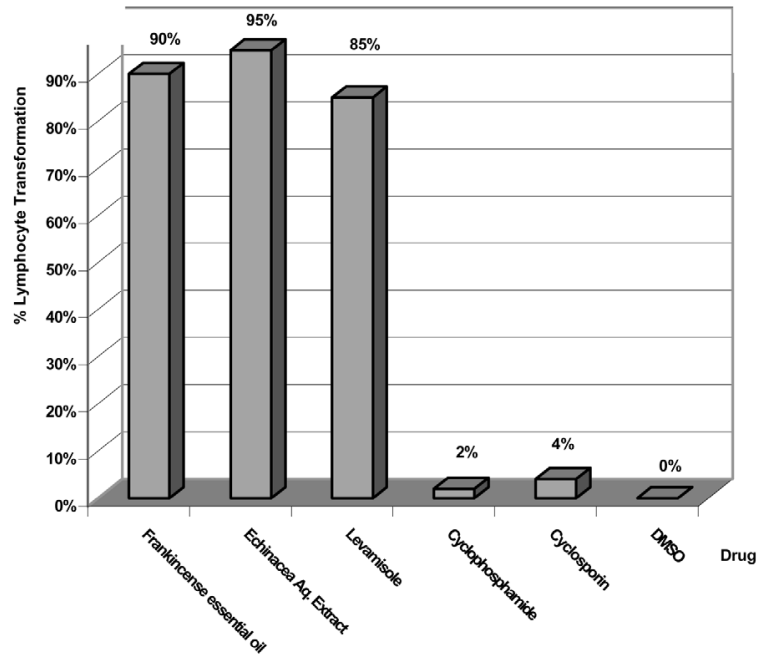

Fig. 2. Results of lympohocyte transformation assay of frankincense essential oil.

lected, dried over anhydrous sodium sulfate and kept in the refrigerator until analysis.

Determination of the physicochemical charateristics and constants

All physicochemical constants of the oil were determined according to BP 1998, optical rotation was determined using ADP 220 polarimeter (Bellingham and Stanely Ltd., Kent, England), refractive index using LEICA ABBE Mark II Refractometer (Leica Inc., Buffalo, NY, USA).

Capillary gas chromatography/ mass spectrometry $(G C / M S)$ analysis

The GC/MS analysis of the oil was carried out at National Research Center, Dokki, Cairo, Egypt on GC/MS Finningan Mat SSQ 7000 with Digital DEC 3000 workstation fitted with a fused silica DB-5 (30 m $\times 0.25 \mathrm{~mm}$ ID, 5\% phenyl methyl polysiloxane) capillary column with helium as carrier gas at a flow rate of $1.6 \mathrm{ml} / \mathrm{min}$, column head pressure 13 Psi. The gas chromatograph was coupled to a mass selective detector (MS) at $70 \mathrm{eV}$ in EI ionization mode. The sample was injected in $1 \mu \mathrm{l}$ volume in splitless mode. The temperature was programmed initially at $60^{\circ} \mathrm{C}$ for $3 \mathrm{~min}$, then increased with a rate of $3^{\circ} \mathrm{C} / \mathrm{min}$ up to $250{ }^{\circ} \mathrm{C}$.
Assessment of the immunomodulatory activity of the oil: lymphocyte blast transformation (mitogensis) assay

The lymphocyte blast transformation (mitogensis) or proliferation assay was applied. The assay was adapted as a test for cell-mediated immunity (Stites, 1987). The cell-mediated immune response was determined in the peripheral blood lymphocytes (PBL) in response to mitogenic stimulation using either phytohaemagglutinin (PHA) or concanavalin A (Con A) as mitogens that stimulate human $\mathrm{T}$ and $\mathrm{B}$ cells but T-cells more vigorously.

\section{A) Separation of peripheral blood lymphocytes (PBL)}

Lymphocytes were separated from peripheral human venous blood by Ficoll/Hypaque gradient technique (Boyum, 1976). For each sample, $5 \mathrm{ml}$ of heparinized blood was diluted with equal volume of Hank's balanced salt solution (HBSS) in a sterile plastic centrifuge tube. $6 \mathrm{ml}$ of diluted blood were carefully overlaid on $4 \mathrm{ml}$ Ficoll/ Hypaque solution gradient without allowing the solution to become mixed by keeping the pipette against the tube wall 5-10 $\mathrm{mm}$ above the fluid meniscus. The tube was centrifuged at $1200 \mathrm{rpm}$ at room temperature. The lymphocytes were localized as a whitish layer on the upper meniscus of the gradient solution. Using a fine pasteur pipette, the zone containing lymphocytes was taken and washed twice in HBSS (10 min at $1200 \mathrm{rpm}$ ). The residue is a buffy coat of polymorphnuclear leucocytes (PMNLs).

\section{B) Lymphocyte transformation assay}

The viable lymphocytes were adjusted to a concentration of $2 \times 10^{6}$ cells $/ \mathrm{ml}$ in RPMI- 1640 medium supplemented with $600 \mu \mathrm{l}$ penicillin, $0.1 \mathrm{ml}$ streptomycin, $1 \%$ glutamine, 25\% HEPES (N-2-Hydroxyethylpiperazine- $\mathrm{N}^{\prime}$-2-ethanesulfonic acid)-buffer, and $20 \%$ foetal calf serum (FCS). The lymphocytes were plated into 96-well tissue culture plates (or Ependorf tubes). $100 \mu \mathrm{l}$ of the volatile oil solution in DMF $(100 \mu \mathrm{l} / \mathrm{ml})$ and $20 \mu \mathrm{g}$ of the mitogen (PHA) were added to each well. Cell cultures were incubated at $37{ }^{\circ} \mathrm{C}$ in $5 \% \mathrm{CO}_{2}$ atmosphere for $72 \mathrm{~h}$, during which the mitogen produces its maximal effect on DNA synthesis. After culture, 
cell films were stained by Giemsa stain and average count of percentage of transformed (proliferated) blasts was determined. Aqueous Echinaceae purpurea extract (Immulone ${ }^{\circledR}$ ) and levamisole $\left(\operatorname{Ketrax}^{\circledR}\right)$ were used as positive control (standard immunostimulant) while cyclophosphamide (Endoxan ${ }^{\circledR}$ ) and cyclosporin (Sandimmune ${ }^{\circledR}$ ) were used as negative control (standard

Abdel wahab S. M., Aboutabl E. A., Elzalabani S. M., Fouad H. A., De Pooter H. L., and El Fallaha B. (1987), The essential oil of olibanum. Planta Med. 53, 382-384.

Adams R. P. (1989), Identification of Essential Oils by Ion Trap Mass Spectroscopy. Academic Press Inc, San Diego, California

Adams R. P. (1995), Identification of Essential Oil Components by Gas Chromatography Mass Spectroscopy. Allured Publ. Corp., Wheaton, Illinois.

Ammar N., Founier G., and El-Deeb S. (1994), The volatile constituents of Boswellia sacra: Frankincense. J. Drug Res.-Cairo 21, 55-58.

Ammon, H. P. T., Singh T., and Safayhi H. (1991), Inhibition of leukotriene $\mathrm{B} 4$ formation in rat peritoneal neutrophils by an ethanolic extract of the gum resin exudate of Boswellia serrata. Planta Med. 57, 203-207.

Boyum A. (1976), Isolation of lymphocytes, granulocytes, and macrophages. Scand. J. Immunol. Suppl. 5, $9-15$.

British Pharmacopeia (1998), (c) Copyright Crown Publishing, London, Vol. II, pp. A138, A139, A171-A173.

Dietrich K. (1900), Analyse der Harze, Balsame und Gummiharze neben ihrer Chemie und Pharmacognosie, Springer Publ., Berlin, p. 262.

Eklund A. M., Berg J. E., and Wahlberg I. (1992), Tobacco chemistry. 73; 4,6,8-trihydroxy-11-capnosene2,10-dione, a new cembrane-derived bicyclic diterpenoid from tobacco. Acta Chem. Scand. 46, 367-371.

Evans, W. C. (1996), Trease \& Evans Pharmacognosy, $14^{\text {th }}$ Edition. WB Saunders Company Ltd., London, p. 289.

Gangwal M. L., and Vardhan D. K. (1995), Antifungal studies of volatile constituents of Boswellia serrata. Asian J. Chem. 7, 675-678.

Guenther E. (1972), The Essential Oils. Vol. IV, Robert E. Krieger Publ. Co., New York, pp. 352-356.

Gupta I., Parihar A., Malhotra P., Singh G. B., Ludtke R., Safayhi H., and Ammon H. P. (1997), Effects of Boswellia serrata gum resin in patients with ulcerative colitis. Eur. J. Med. Res. 2, 37-43.

Gupta I., Gupta V., Parihar A., Gupta S., Ludtke R., Safayhi H., and Ammon H. P. (1998), Effects of Boswellia serrata gum resin in patients with bronchial asthma: results of a double-blind, placebo-controlled, 6-week clinical study. Eur. J. Med. Res. 3, 511-514. immunosuppressant) $100 \mu \mathrm{g} / \mathrm{ml}$ of each drug in DMSO.

\section{Acknowledgement}

Dr. Samia A. Hawas, Professor of Clinical Immunology, Faculty of Medicine, Mansoura University, Egypt, is very much thanked for performing the lymphocyte transformation assay.

Hayashi S., Amemori H., Kameoka H., and Hanafusa M. (1998), Comparison of volatile components from olibanum from various countries. J. Ess. Oil Res. 10, $25-30$.

Lawless J. (1996), The Illustrated Encyclopaedia of Essential Oils, Element Books, London, p. 97.

Leung, A. Y., and Foster S. (1996), Encyclopaedia of Common Natural Ingredients Used in Food, Drugs, and Cosmetics. 2nd Ed., John Wiley and Sons, New York, pp. 389-391.

Maria L. B., and Stephen H. (1997), A preliminary study of the effect of essential oils on skeletal and smooth muscles in vitro. J. Ethnopharmacol. 58, 183-187.

Maupetit P. (1984/1985), New constituents in olibanum resinoid and essential oils. Perfumer and Flavorist 9, 19-20, 22, 24-26, 28, 30, 32-34, 36-37.

Nores M. M., Courrèges M., Benencia F., and Coulombie F. C. (1997), Immunomodulatory activities of $\mathrm{Ce}$ drela lilloi and Trichilia elegans leaf extracts. J. Ethnopharmacol. 55, 99-106.

Obermann H. (1978), Monoterpensäuren als Spurenkomponenten in Olibanumöl. Dragoco Rep. 3, 55-58.

Ohloff G. (1994), Scent and Fragrances (translated by Wilhelm Pickenhagen and Brian M. Lawrence). Springer Publ., Berlin, Heidelberg, p. 188.

Puri A., Saxena R., Saxena R. P., and Saxena K. C. (1993), Immunostimulant agents from Andrographis paniculata. J. Nat. Prod. 56, 995-999.

Rashid M. A., Gustafson K. R., and Boyd M. R. (2000), HIV-Inhibitory cembrane derivatives from a Philippines collection of the soft coral Lobophytum sp. J. Nat. Prod. 63, 531-533.

Ryman D. (1997), Aromatherapy, the Encyclopaedia of Plants and Oils. Judy Piatkus Publishers Ltd., London, pp. $101-102$.

Safayhi H., Mack T., Sabieraj J., Anazodo M. I., Subramanian L. R., and Ammon H. P. T. (1992), Boswellic acids: Novel, specific, nonredox inhibitors of 5-lipoxygenase. J. Pharmacol. Exp. Therap. 261, 1143-1146.

Safayhi H., Sailer E. R., and Ammon H. P. T. (1995), Mechanism of 5-lipoxygenase inhibition by acetyl-11keto-Boswellic acid. Mol. Pharmacol. 47, 1212-1216.

Sairam M., Sharma G. I., Kumar D., and Selvamurthy W. (1997), Immunomodulatory effects of NIM-76, a volatile fraction from Neem oil. J. Ethnopharmacol. 55, 133-139. 
Sharma M. L., Khajuria A., Kaul A., Singh S., Singh G. B., and Atal C. K. (1988), Effect of salai guggal exBoswellia serrata on cellular and humoral immune responses and leucocyte migration. Agents Actions 24, $161-164$.

Singh G. B., and Atal C. K. (1986), Pharmacology of an extract of salai guggal ex-Boswellia serrata, a new non-steroidal anti-inflammatory agent. Agents Actions 18, 407-412.

Stites D. P. (1987), Clinical laboratory methods for detection of cellular immune function, In: Basic and Clinical Immunology, $6^{\text {th }}$ edition. Appleton Lange Norwalk, Connecticut, Los Atlos, California, pp. 285-286.
Strappaghetti G., Corsano S., Craveiro A., and Proietti G. (1982); Constituents of essential oil of Boswellia frereana. Phytochemistry 21, 2114-2115.

Tschrich A., and Stock E. (1935), Die Harze, Gebrüder Bornträger, Berlin, p. 239.

Verghese J. (1988), Olibanum in focus. Perfumer and Flavorist 13, 2-11.

Wallis T. E. (1967), Text Book of Pharmacognosy, $5^{\text {th }}$ Edition. J. \& A. Churchill Ltd, London, pp. 500501.

Yates R. L., and Wenninger J. A. (1970), Constituents of olibanum oil: Sesquiterpene hydrocarbons. J. Assoc. Anal. Chem. 53, 941-948. 